\title{
A Monte Carlo study of cosmological parameter estimators from galaxy cluster number counts
}

\author{
Mariana Penna-Lima ${ }^{1}$, Martín Makler $^{2}$ and Carlos A. Wuensche ${ }^{1}$ \\ ${ }^{1}$ Divisão de Astrofísica, Instituto Nacional de Pesquisas Espaciais, \\ Av. dos Astronautas 1758, 12227-010, São José dos Campos, Brazil \\ email: mariana.lima@inpe.br \\ ${ }^{2}$ Centro Brasileiro de Pesquisas Físicas, \\ Rua Dr. Xavier Sigaud 150 22290-180, Rio de Janeiro, Brasil \\ email: martin@cbpf.br \\ email: ca.wuensche@inpe.br
}

\begin{abstract}
Models for galaxy clusters abundance and their spatial distribution are sensitive to cosmological parameters. Present and future surveys will provide high-redshift sample of clusters, such as Dark Energy Survey $(z \leqslant 1.3)$, making cluster number counts one of the most promising cosmological probes. In the literature, some cosmological analyses are carried out using small cluster catalogs (tens to hundreds), like in Sunyaev-Zel'dovich (SZ) surveys. However, it is not guaranteed that maximum likelihood estimators of cosmological parameters are unbiased in this scenario. In this work we study different estimators of the cold dark matter density parameter $\Omega_{c}, \sigma_{8}$ and the dark energy equation of state parameter $w_{0}$ obtained from cluster abundance. Using an unbinned likelihood for cluster number counts and the Monte Carlo approach, we determine the presence of bias and how it varies with the size of the sample. Our fiducial models are based on the South Pole Telescope (SPT). We show that the biases from SZ estimators do not go away with increasing sample sizes and they may become the dominant source of error for an all sky survey at the SPT sensitivity.
\end{abstract}

Keywords. (cosmology:) cosmological parameters, galaxies: clusters: general

\section{Introduction}

Galaxy cluster abundance and their spatial distribution as functions of redshift and mass provide strong constraints on the matter density parameter $\Omega_{m}$ and the amplitude $\sigma_{8}$ of the power spectrum. For high redshift surveys $(z>1.0)$, clusters are also powerful tools to study dark energy (DE) since they depend on the linear growth of density perturbations and the comoving volume (see Allen et al. 2011 and references therein).

Usually the cluster likelihood function is built taking into account the following error sources: the uncertainties of the mass-observable relations and the photometric redshifts, sample-variance and shot-noise. However, in general, it is assumed that the cosmological parameter estimators themselves do not induce any extra uncertainty.

The Maximum Likelihood (ML) method is extensively used to estimate the values of parameters given a data set. ML estimators are usually consistent, i.e., the expected value of a parameter tends to its true value when the size of the data sample $(n)$ is sufficiently large, and asymptotically efficient, i.e., the variance of the estimator attains the minimum variance bound as $n \rightarrow \infty$. However, ML estimators are not necessarily unbiased. 
In this work, we study some estimators of $\Omega_{c}, \sigma_{8}$ and $w_{0}$ in the context of SZ surveys, specifically from the SPT. For the redshift and mass ranges of SPT catalogs, sample variance is negligible. Therefore, we build the likelihood only for the cluster number counts and do not include the spatial clustering.

\section{Methodology}

The likelihood for cluster number counts is

$$
\ln \mathcal{L}\left(\left\{\theta_{j}\right\},\left\{\xi_{i}, z_{i}\right\}\right)=\sum_{i=1}^{n} \ln \left(\frac{d^{2} N\left(\xi_{i}, z_{i},\left\{\theta_{j}\right\}\right)}{d z d \xi}\right)-N\left(\left\{\theta_{j}\right\}\right)-\ln n !,
$$

where $n$ is the total number of clusters, $\xi$ is the detection significance (Vanderlinde et al. 2010 and Benson et al. 2013) and

$$
\frac{d^{2} N\left(\xi_{i}, z_{i},\left\{\theta_{j}\right\}\right)}{d z d \xi}=\int d \ln M \int d \zeta \frac{d^{2} N\left(M, z_{i},\left\{\theta_{j}\right\}\right)}{d z d \ln M} P\left(\xi_{i} \mid \zeta\right) P(\ln \zeta \mid \ln M) .
$$

The normalization factor $N\left(\left\{\theta_{j}\right\}\right)$ is the expected number of clusters with $0.3 \leqslant z \leqslant 1.1$ and $\xi_{\min } \geqslant 5$. We assume that the catalog is complete above this threshold and in the entire redshift interval.

Estimators for a given set of parameters $\left(\left\{\hat{\theta}_{j}\right\}\right)$ are obtained by minimizing $-2 \ln \mathcal{L}\left(\left\{\theta_{j}\right\}\right.$, $\left.\left\{\xi_{i}, z_{i}\right\}\right)$ with respect to these parameters. The bias of $\hat{\theta}_{j}$ is defined as

$$
b_{\hat{\theta}_{j}}=\left\langle\hat{\theta}_{j}\right\rangle-\theta_{j}^{0},
$$

where $\left\langle\hat{\theta}_{j}\right\rangle$ is the expected value and $\theta_{j}^{0}$ is the true value (fiducial). We cannot compute $\left\langle\hat{\theta}_{j}\right\rangle$ analytically. Therefore, we use the Monte Carlo (MC) method, i.e., we generate a set of realizations of the input fiducial model and compute the best-fitting value of $\hat{\theta}_{j}$ for each realization. Then we compute the expected value using as an estimate

$$
\left\langle\hat{\theta}_{j}\right\rangle \approx \overline{\hat{\theta}}_{j} \pm \sigma\left(\overline{\hat{\theta}}_{j}\right), \quad \overline{\hat{\theta}}_{j}=\sum_{l=1}^{m} \frac{\hat{\theta}_{j l}}{m}, \quad \sigma\left(\overline{\hat{\theta}}_{j}\right)=\frac{\sigma\left(\hat{\theta}_{j}\right)}{\sqrt{m}},
$$

where $m$ is the number of realizations and $\hat{\theta}_{j l}$ is the best-fitting value for the l-th realization. In cases where $b_{\hat{\theta}_{j}} \neq 0$, the significance of this bias is ensured computing $\sigma\left(\overline{\hat{\theta}}_{j}\right)$. In particular for our purposes, it was necessary to generate 10,000 realizations for each choice of the fiducial model.

To obtain a realization, we first randomly generate the number of objects $n$ using $N\left(\left\{\theta_{j}^{0}\right\}\right)$ as the mean of a Poisson distribution. Then, we apply the inverse transform sampling (ITS): giving the probability distribution of finding a cluster with detection significance greater than $\xi_{\text {min }}$ and in $[z, z+d z]$, i.e.,

$$
\mathcal{P}(z) d z=\frac{1}{N\left(\left\{\theta_{j}\right\}\right)}\left(\frac{d N}{d z}\right) d z
$$

we define the cumulative

$$
f(z)=\int_{0}^{z} \mathcal{P}\left(z^{\prime}\right) d z^{\prime} .
$$

Given that $f(z)$ is a monotonically increasing function, whose image is $[0,1]$, and a random variable with uniform distribution over $[0,1]$, we generate $n$ random numbers $\left\{u_{i}\right\}$ from a uniform distribution and invert Eq. 2.6 obtaining $z(f)$ and, therefore, 

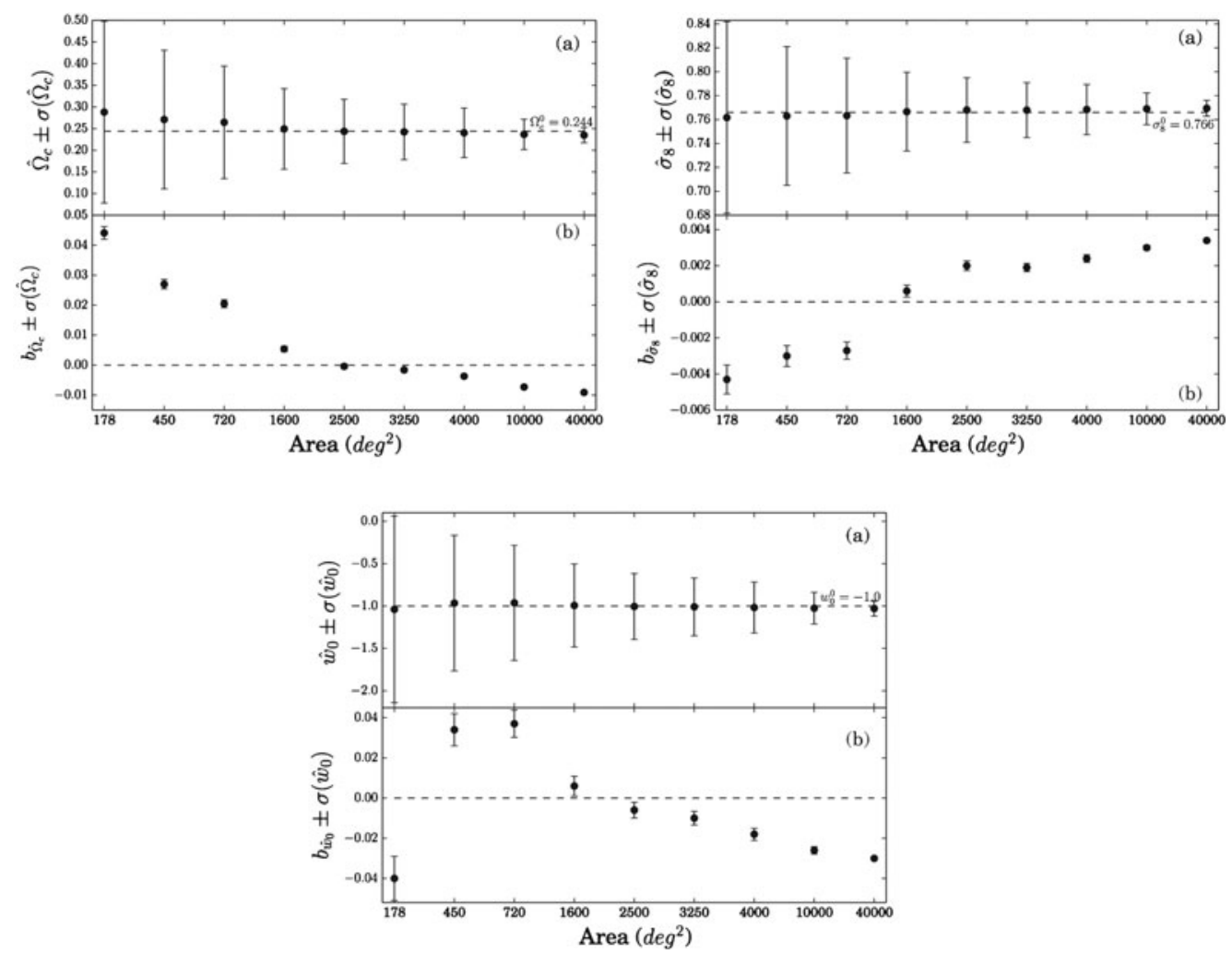

Figure 1. The expected values of the estimators (dots) are displayed with the error bars of the estimators (a) and of their means (b) for different survey areas. This analysis was done in the three-dimensional parametric space $\left(\Omega_{c}, \sigma_{8}, w_{0}\right)$, keeping all other parameters fixed.

$\left\{z_{i}\right\}=\left\{z\left(u_{i}\right)\right\}$. Analogously, we obtain a value $\xi_{i}$ from the conditional distribution of obtaining a cluster with detection significance in the range $\xi$ and $\xi+d \xi$ given $z_{i}$,

$$
P\left(\xi \mid z_{i}\right)=\frac{P\left(\xi, z_{i}\right)}{\mathcal{P}\left(z_{i}\right)} .
$$

Then, we apply the ITS method, given the cumulative $f\left(\xi \mid z_{i}\right)$, obtaining $\left\{\xi_{i}, z_{i}\right\}$.

\section{Results and Concluding Remarks}

The fiducial model corresponds to the best-fit values obtained in Benson et al. (2013) by combining cluster abundance, Hubble parameter and Big-Bang nucleosynthesis measurements, assuming a $\Lambda \mathrm{CDM}$ model: $\Omega_{c}=0.244, \Omega_{b}=0.0405, H_{0}=73.9, n_{s}=0.966$, $\sigma_{8}=0.766, w_{0}=-1, A_{S Z}=5.31, B_{S Z}=1.39, C_{S Z}=0.9$ and $D_{S Z}=0.21$. Giving this fiducial model and varying only the angular survey area, we apply the MC approach and compute Eqs. (2.3) and (2.4).

Fig. 1 shows that almost all estimators of $\hat{\Omega}_{c}, \hat{\sigma}_{8}$ and $\hat{w}_{0}$ are biased. Both $b_{\hat{\theta}}$ and the relative biases, $B_{\hat{\theta}_{j}} \equiv b_{\hat{\theta}_{j}} / \sigma\left(\hat{\theta}_{j}\right)$, depend on the survey area (sample size). $B_{\hat{\theta}}$ for $\Delta \Omega=10,000$ and $40,000 \mathrm{deg}^{2}$ are not negligible, namely, for the last, $B_{\hat{\Omega}_{c}}=52 \%$, $B_{\hat{\sigma}_{8}}=51 \%$ and $B_{\hat{w}_{0}}=33 \%$. These results in the full-sky limit evince that cluster abundance estimators using SPT $\xi$-mass relation are not consistent. 
Besides the results presented, we performed a comprehensive study in Penna-Lima et al. (2014), considering, for example, photometric redshift uncertainty and a combined likelihood of clusters and the distance priors of the cosmic microwave background. To perform this work, we developed fast, accurate, and adaptable codes for cluster counts in the framework of the Numerical Cosmology library (www.nongnu.org/numcosmo).

\section{References}

Allen, S. W., Evrard, A. E., \& Mantz, A. B. 2011, ARAA, 49, 409

Benson, B. A., de Haan, T., Dudley, J. P., Reichardt, C. L., Aird, K., et al. 2013, ApJ, 763, 147

Penna-Lima, M., Makler, M., \& Wuensche, C. A. 2014, JCAP, 5, 39

Vanderlinde, K., Crawford, T. M., de Haan, T., Dudley, J., Shaw, L., et al. 2010, ApJ, 722, 1180 